\title{
Quantitative absorption spectroscopy of nano-objects
}

\author{
Pascal Berto ${ }^{1}{ }^{*}$ Esteban Bermúdez Ureña ${ }^{2}$, Pierre Bon ${ }^{1}$, \\ Romain Quidant $^{2,3}$, Hervé Rigneault ${ }^{1}$, and Guillaume Baffou ${ }^{1 \dagger}$ \\ ${ }^{1}$ Aix Marseille Universit, CNRS, Ecole Centrale Marseille, Institut Fresnel, 13013 Marseille, France \\ ${ }^{2}$ ICFO - Institut de Ciencies Fotoniques, Mediterranean Technology Park, 08860 Castelldefels (Barcelona), Spain and \\ ${ }^{3}$ ICREA - Institució Catalana de Recerca i Estudis Avançats, 08010 Barcelona, Spain
}

(Dated: September 29, 2012)

\begin{abstract}
We report on an optical microscopy technique capable of directly measuring the absolute absorption cross section of individual nanoparticles. It relies on the thermally induced variation of the refractive index of the surrounding medium subsequent to light absorption by the nanoparticle. The techniques is illustrated on gold nanoparticles featuring a well-defined plasmonic resonance. Different plasmonic modes were evidenced and quantified. The simplicity and rapidity of the measurements make it possible to investigate absorption resonances of absorbing nano- and micro-structures in a reasonable time frame. The experimental approach is furthermore simply based on the use of a wavefront analyzer, which is straightforward to implement on any conventional microscope.
\end{abstract}

\section{INTRODUCTION}

The likelihood of interaction between light and a nanoparticle (NP) can be quantified by different optical cross sections. The absorption cross section (ACS) $\sigma_{\text {abs }}$ quantifies the ability of the NP to absorb an incident photon, while the scattering cross section $\sigma_{\text {sca }}$ refers to the ability of the NP to scatter a photon. These cross sections are defined such that the power $P_{\text {abs }}$ absorbed by the NP and the power $P_{\text {sca }}$ scattered by the NP simply read $P_{\mathrm{abs}}=\sigma_{\mathrm{abs}} I$ and $P_{\mathrm{sca}}=\sigma_{\mathrm{sca}} I$, where $I$ is the irradiance (power per unit area) of the incoming light. The total (or extinction) cross section is $\sigma_{\text {ext }}=\sigma_{\text {sca }}+\sigma_{\text {abs }}$. Depending on the nature of the NP, its morphology, and the excitation wavelength, the relative efficiencies of absorption and scattering can be very different from one NP to another.

Light absorption by a nano-object is often considered as a source of energy loss since it usually corresponds to a conversion of light into heat. However, since recent developments in thermo-plasmonics, ${ }^{1}$ this transduction pathway has been promoted since it makes it possible to use a metal NP as a nano-source of heat, one of the most fundamental tool in nature. ${ }^{2}$ Many emerging research areas and applications are now based on photothermal processes of noble metal NPs. In this field, the knowledge of the maximum absorption wavelength and the corresponding ACS are important to optimize heat generation. However, measuring the absorption cross section of a nano-object remains a difficult task, mainly because the information of interest is no longer radiated and detectable in the far field. Several techniques have been developed recently to estimate ACSs of metal NPs. ${ }^{3-5}$ However, they present major limitations: i) They rely on the assumption that ACS can be assimilated to extinction cross section. This assumption is valid only for NPs much smaller than the illumination wavelength. ii) They do not lead to the absolute value of the ACS. iii) They use a complex instrumentation. iv) They are relatively slow.

We report here on an optical microscopy technique

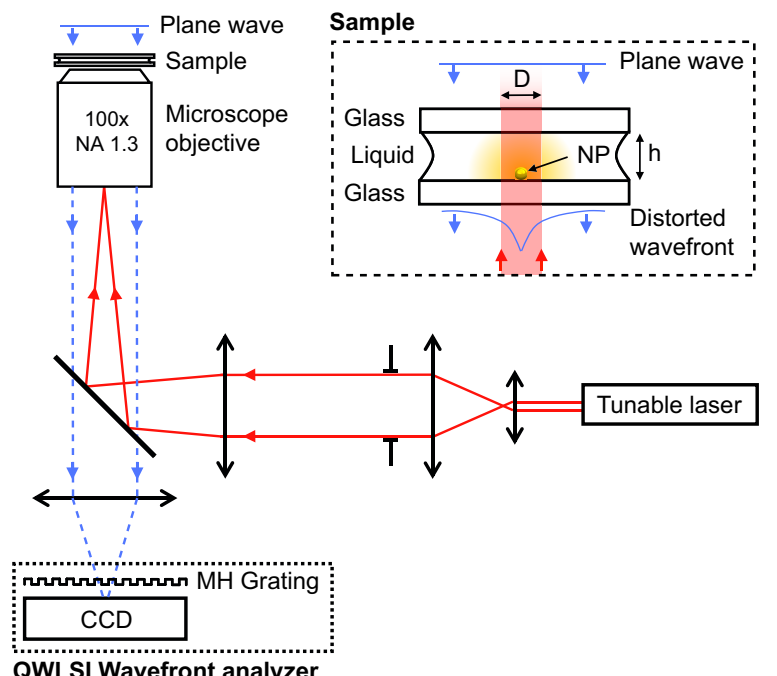

FIG. 1. Experimental configuration used to perform quantitative absorption spectroscopy measurements. The inset illustrates the wavefront distortion thermally induced by the heated NP.

able to quantitatively and rapidly measure the ACS of absorbing nano-structures, whatever their nature, their size and their morphology. After introducing and describing the principle of the technique, we illustrate its capabilities first with an ensemble measurement on a regular pattern of lithographic gold structures, and then with isolated nanostructures. Then, we discuss the sensitivity of the measurements and explain how to optimize it. A final part is dedicated to make a comparison with previous related techniques reported in the literature. 


\section{EXPERIMENTAL TECHNIQUE}

We recently introduced an optical microscopy technique able to map the distributions of temperature and heat power density around metal nanostructures. ${ }^{6}$ This technique is based on quadriwave lateral shearing interferometry (QWLSI). Our present work follows this development and utilizes a similar experimental setup, which is presented in Fig. 1, to achieve quantitative absorption spectroscopy. The principle consists in probing the thermally induced distortion of a planar optical wavefront passing through the medium of interest. The wavefront distortion is due to light absorption by the NP that generates a temperature increase and a subsequent variation of the refractive index of the surrounding medium. The samples we used consist of lithographic gold nanostructures lying on a planar glass substrate. A drop of glycerol was deposited on this substrate and an additional glass cover slip was pressed on top of the sample to end up with a sandwich-like structure (inset of Fig. 1). The use of a liquid as the surrounding medium ensures a substantial thermally induced variation of the refractive index even for temperature increases as small as $1 \mathrm{~K}$. The sample is illuminated from the top by a collimated broad-band light emitting diode $\left(\lambda_{0}=600 \pm 50 \mathrm{~nm}\right)$. The distorted wavefront profile of this probe beam is then imaged using the QWLSI wavefront sensor. More precisely, a modified Hartmann diffraction grating ${ }^{7}$ produces an interferogram on a CCD camera that can be processed to quantitatively retrieve the optical wavefront profile of the probe beam. ${ }^{6}$ In parallel, a CW Ti:Sapphire laser is used to heat the light absorbing metal structures. The wavelength of this infrared (IR) laser can be tuned from $\lambda_{0}=720$ up to 950 $\mathrm{nm}$. We designed the gold nanostructures to adjust their plasmonic resonances in this wavelength range. To perform an image, a first reference interferogram is recorded without heating (IR laser turned off) from which a reference wavefront profile is computed. Then, the IR laser is turned on to heat the structures and a second interferogram is recorded which yields a second wavefront profile. From the subtraction of these two images, the distribution of the optical path difference (OPD) through the sample experienced by the probe beam is retrieved.

In the case of a point-like NP, the thermally induced OPD can be easily modeled theoretically. In such an ideal case, the temperature profile in the surrounding liquid is simply given by:

$$
T(r)=T_{\infty}+\frac{\sigma_{\mathrm{abs}} I}{4 \pi \bar{\kappa} r}
$$

where $r$ is the radial coordinate, $T_{\infty}$ the ambient temperature, $I$ the irradiance of the illumination and $\bar{\kappa}=$ $\left(\kappa_{1}+\kappa_{2}\right) / 2$ the average of the thermal conductivities of the surrounding liquid and the substrate. Considering the average thermal conductivity $\bar{\kappa}$ is not an approximation. It is the exact solution of the problem, which can be derived using the image method with a source of heat localized right at the interface. ${ }^{8}$ An analytical expression of this associated distorted wavefront can be easily derived if one considers that the refractive index of glycerol varies linearly with the temperature, $\delta n=\beta\left(T-T_{\infty}\right)$. By integrating $\delta n$ over the liquid height we find that the OPD $\delta \ell$ experienced by the beam reads:

$$
\begin{aligned}
& \delta \ell(r)=\frac{P}{4 \pi \bar{\kappa}} \beta \ln \left(h / r+\sqrt{1+(h / r)^{2}}\right) \\
& \delta \ell(r)=P G_{\ell}(r)
\end{aligned}
$$

where $P=\sigma_{\text {abs }} I$. Eq. 3 defines the Green's function $G_{\ell}$ discussed further in this work. Eq. 2 can be directly used to quantitatively estimate the delivered power $\sigma_{\text {abs }}$ of a point-like NP since the liquid thickness $h$ and the irradiance $I$ can be easily estimated, and the liquid parameters $\bar{\kappa}$ and $\beta$ are presumably known. One just has to fit the radial profile of the OPD with $\sigma_{\mathrm{abs}}$ as the unique fitting parameter.

This technique is not limited to retrieve the ACS of point-like NPs. In the case of an extended absorbing structure of any shape and size, the formalism can be refined to retrieve as well the overall ACS. The OPD profile in the surrounding medium appears in this case as a convolution between the heat power density $p(x, y)$ of the sample and the Green's function $G_{\ell}$ (Eq. 3):

$$
\delta \ell(\mathbf{r})=\left[p \otimes G_{\ell}\right](\mathbf{r})
$$

Hence, the distribution of $p(x, y)$ can be retrieved by a deconvolution procedure. Once $p(x, y)$ is known, the overall ACS of the extended pattern reads

$$
\sigma_{\text {abs }}=\frac{1}{I} \int p(x, y) \mathrm{d} x \mathrm{~d} y
$$

\section{RESULTS AND DISCUSSION}

We shall now illustrate the technique by performing quantitative absorption spectroscopy in the two above mentioned cases: First an extended distribution of nanostructures and then a single isolated NP.

Figure 2 presents a series of measurements performed on an array of identical lithographic gold dimer structures. Each dimer consists of two rods of length $L$ and width $50 \mathrm{~nm}$ separated by a gap of $30 \mathrm{~nm}$ (Fig. 2a). Several arrays characterized by different rod lengths $L$ have been fabricated and investigated. The central part of the array was illuminated by a uniform circular beam of diameter $D=14 \mu \mathrm{m}$ to generate optical absorption and heat. The liquid height was set to $h=1.0 \mathrm{~mm}$. Measurements were performed at various wavelengths close to the resonance of the nanostructures in order to retrieve the absorption spectra. Figure 2 a presents a SEM image of an array of gold dimers with $L=320 \mathrm{~nm}$. The size of the illuminated area is represented by a circle. At each wavelength, the distribution of heat source density $p(x, y)$ (Fig. 2c) is retrieved from the distribution of the thermal-induced OPD (Fig. 2b) using Eq. 4 and a deconvolution algorithm. The total absorbed power $P$ 
a
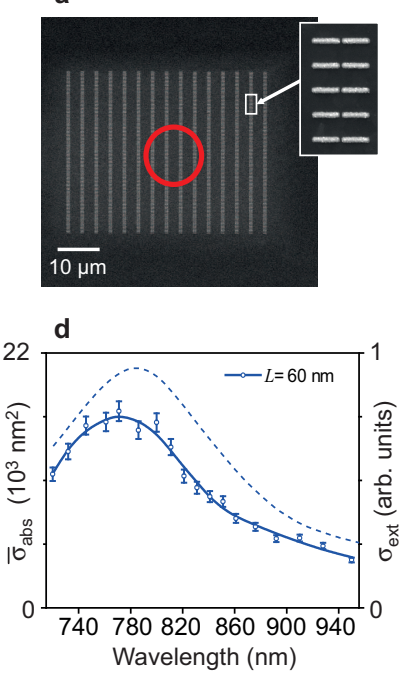

b
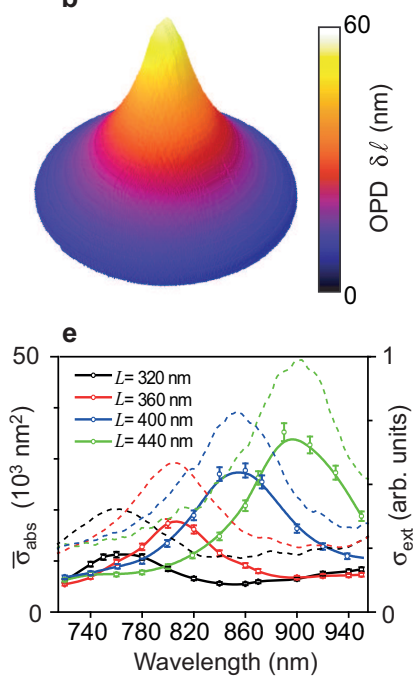
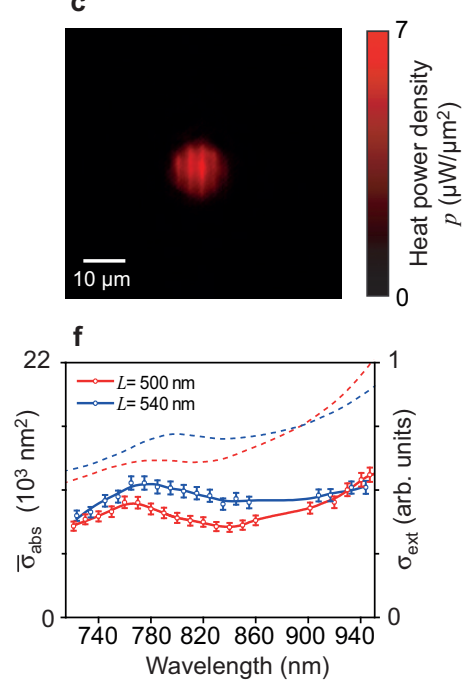

FIG. 2. a) Scanning Electron Microscopy (SEM) image of an array of lithographic gold dimers (rod length $L=320 \mathrm{~nm}$ ) made by e-beam lithography. b) Measured distribution of the thermally-induced optical path difference (OPD) when heating the array with a uniform circular beam at $\lambda_{0}=750 \mathrm{~nm}$. c) Associated heat power density computed from image b. d) Experimental absorption spectrum (solid line) of dimers, along with the associated extinction spectra (dash line) measured using standard extinction spectroscopy. The resonance corresponds to the plasmonic dipolar mode of the structure. e) Same as image d for 4 sizes of dimers corresponding to the $3 \lambda / 2$ plasmonic mode. f) Same as image $d$ for 2 sizes of dimers corresponding to the $5 \lambda / 2$ mode.

is estimated quantitatively by spatially integrating the heat power density (Eq. 5). Since the extended structure is composed of identical NPs, we can get a first estimation of the single-NP ACS by considering the quantity $\bar{\sigma}_{\text {abs }}=P /(I N)$ where $N$ is the estimated number of NPs in the illuminated area. This procedure was repeated at various wavelengths to get a quantitative NP absorption spectra. Different nanostructures with different sizes have been investigated as represented in Fig. 2d. In this series of measurements, we wish to illustrate that different absorption modes can be identified. The resonance observed in Fig. 2d corresponds to the dipolar resonance of the structure. When increasing the length $L$ of the structure, this resonance red-shifts and disappears far in the IR while a second resonance arrives from the visible range of the spectrum. This resonance corresponds to a $3 \lambda_{0} / 2$ plasmonic mode of the structure, ${ }^{9}$ as represented in Fig. 2e for different $L$. Finally, while increasing even more the length of the structure $L$, the weak $5 \lambda_{0} / 2$ resonance can also be observed and quantified (Fig. 2f). In all the measured absorption spectra, we also plot the associated extinction spectra measured using conventional means. In the case of the dipolar resonance, a slight shift of $20 \mathrm{~nm}$ between the absorption and the extinction spectra is observed, which is the expected behavior according to the literature. ${ }^{10}$

We shall now describe absorption spectroscopy performed on single NPs. Figure 3 presents a series of measurements performed on single dimer nanostructures (Fig. 3a), as the ones investigated in the previous paragraph. For this measurement, the liquid thickness was set to $h=7 \mu \mathrm{m}$ and the illumination diameter $D=6.2 \mu \mathrm{m}$. This time, the OPD distribution is much more confined as observed in (Fig. 3b). As mentioned before, the estimation of the ACS is much simpler for single NPs since the computation of the heat power density distribution (using a deconvolution procedure) is not required. The heat power can be directly retrieved from the OPD image. The OPD radial profile is simply fitted using Eq. 2 with the delivered power $P$ as the only fitting parameter. A perfect matching between Eq. 2 and the OPD radial profile is usually observed as shown in Fig. 3c. Then, the ACS is simply given by $\sigma_{\text {abs }}=P / I$. The procedure was repeated for various wavelengths, which gave the absorption spectra represented in Fig. 3d. In this figure, the absorption spectra obtained in the previous section from an array of NPs is also represented and is, as expected, identical. The measured extinction spectra is also plotted, showing the same resonance wavelength. At the current stage of its development, ACSs as small as $c a .10^{3} \mathrm{~nm}^{2}$ can be measured. Using a liquid with a higher refractive index dependence on temperature $\beta$ and a CCD camera with a faster readout (in order to collect more photons) could improve the sensitivity of the method in the future.

We wish to underline a limitation that can occur in specific conditions while performing absorption measurements. While investigating single NPs, it may happen that the energy absorbed by the illuminated surrounding liquid becomes more important than the energy absorbed by the NP itself. In this case, the temperature increase is also due to liquid heating and the wavefront distortion is 
a
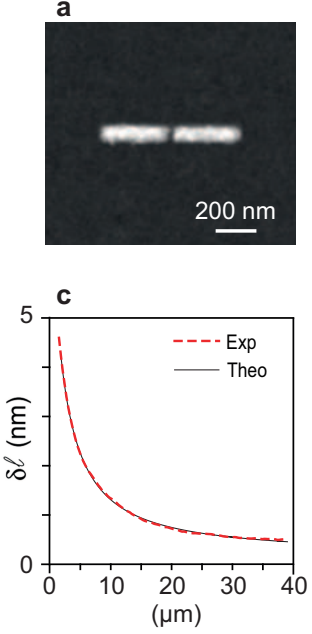

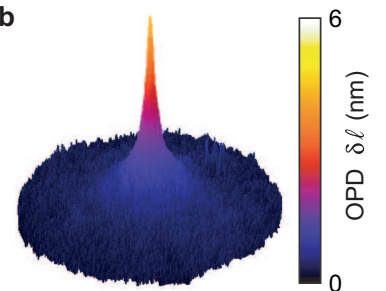

d

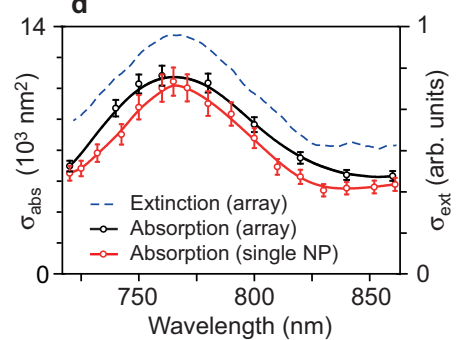

FIG. 3. a) SEM image of a gold dimer $(L=320 \mathrm{~nm})$ made by e-beam lithography. b) Measured distribution of the thermally-induced OPD when heating a single dimer with a uniform circular beam at $\lambda_{0}=740 \mathrm{~nm}$. c) Radial profile of the OPD distribution in image $\mathrm{b}$ in good agreement with the fit function (Eq. 2). d) NP absorption spectra (solid lines) measured on a single dimer and averaged on an array of dimers along with the measured extinction spectrum (dash line).

no longer a specific signature of the NP. Absorption spectra of different liquids were measured using a standard photospectrometer. Results are represented in Fig. 4a and show that strong absorption usually occur after 850 $\mathrm{nm}$ for usual solvents such as water, glycerol and ethanol. The occurrence of such a side effect can be clearly identified on the OPD image as illustrated in Fig. 4b where two OPD profiles are represented: A first OPD profile taken at $\lambda_{0}=720 \mathrm{~nm}$ and a second at $\lambda_{0}=950 \mathrm{~nm}$ where liquid absorption dominates. In the latter case, the heated liquid layer creates a linear slope on the OPD profile at high radial coordinate $r$ that cannot be fitted using Eq. 2 . This abnormal slope is a sign of liquid heating. Hence, absorption spectroscopy in the IR would require the use of a non-absorbing solvent. The importance of such an effect can be predicted by estimating the order of magnitudes of the powers absorbed by the NP itself $P_{\mathrm{NP}}$ and by the liquid layer $P_{\text {liq }}$. It yields:

$$
\xi=\frac{P_{\mathrm{liq}}}{P_{\mathrm{NP}}}=\frac{S I\left(1-e^{\alpha h}\right)}{\sigma_{\mathrm{abs}} I} \simeq \frac{\alpha S h}{\sigma_{\mathrm{abs}}}
$$

where $S=\pi D^{2} / 4, D$ is the diameter of the beam (assumed to be cylindrical over the liquid height) and $\alpha$ the extinction coefficient of the liquid. According to this expression, and as expected, the influence of the liquid heating can be minimized $(\xi \ll 1)$ by reducing the height $h$ of the liquid or the diameter $D$ of the incoming beam. In the experiments reported in Fig. 2, $\alpha \simeq 0.018$ $\mathrm{cm}^{-1}$ and $\bar{\sigma}_{\text {abs }}=10^{3} \mathrm{~nm}^{2}$ at $\lambda_{0}=800 \mathrm{~nm}$. Given that 152 NPs where illuminated it yields $\xi \simeq 0.02$, which is weak enough. On an isolated NP, like in the experiments
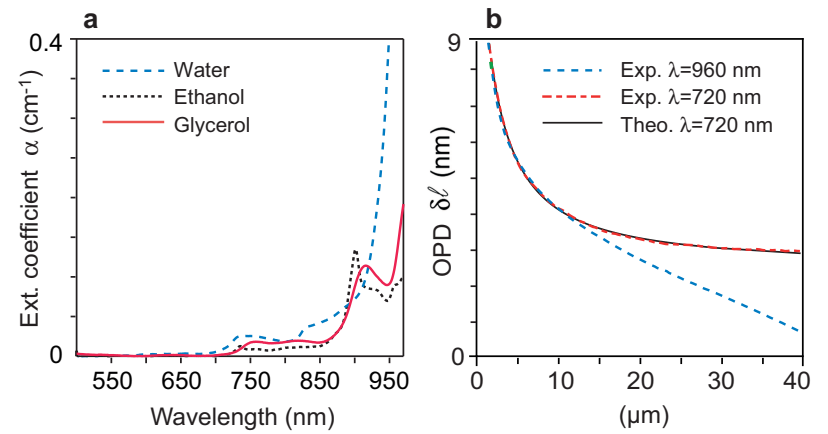

FIG. 4. a) Absorption spectra of various liquids. b) Radial profile of the OPD distribution around a single gold NP at $\lambda_{0}=720 \mathrm{~nm}$ and $\lambda_{0}=960 \mathrm{~nm}(D=6.2 \mu \mathrm{m}$ et $h=7 \mu \mathrm{m})$. In the second case, the heating of the liquid is responsible of an abnormal linear slope at large distances.

reported in Fig. 3, such values of $D$ and $h$ would have led to $\xi \simeq 2.7>1$. This is why we chose to reduce the height of the liquid layer down to $h=7 \mu \mathrm{m}$ and the beam diameter to $D=6.2 \mu \mathrm{m}$. In such conditions, $\xi=0.004 \ll 1$ at $\lambda_{0}=800 \mathrm{~nm}$ and $\xi$ remained small up to $\lambda_{0}=850 \mathrm{~nm}$.

We wish finally to compare our technique to the other reported techniques aimed at measuring ACSs, in order to clarify the advantages and the drawbacks of each approaches.

Let us first depict an important class of photothermal imaging (PTI) techniques, introduced in a pioneer article by Orrit, Lounis and coworkers in 2002. ${ }^{11}$ In this article, the authors report on a method able to detect single nanoparticles smaller than $3 \mathrm{~nm}$ by optically heating them. Such an achievement combined high-frequency modulation and a polarization interference contrast (DIC) measurement. In 2004, Berciaud, Lounis and coworkers made this approach even simpler and more sensitive by implementing a heterodyne detection and measuring a modulated scattered intensity. ${ }^{12}$ This improved version of the technique was then used by the same group to qualitatively measure absorption spectra of nano-objects such as metal NPs, carbon nanotubes ${ }^{13}$ or semiconductor nano-crystals. ${ }^{14}$ In 2010 , Gaiduk, Orrit and coworkers optimized the sensitivity of this heterodyne technique and managed to detect single dye molecules, with an ACS as weak as $1 \mathrm{~nm}^{2}{ }^{5}$

This series of reported experiments is closely related to our QWLSI technique since it is based on the same physics: a photothermal induced variation of the refractive index surrounding the absorbing structure. The fundamental differences is that we do not modulate the heating and we do not perform the same kind of measurements: While the PTI techniques measure either a modulated scattered intensity, ${ }^{5,13}$ or a combination of both the modulated intensity and phase gradient by DIC, ${ }^{11}$ the QWLSI technique measures a quantitative $2 \mathrm{D}$ phase image. ${ }^{6}$ Minding the phase and not the scattered inten- 
sity leads us to the possibility to quantitatively estimate the heat power delivered by the NP. Indeed, in the case of the PTI technique, a direct estimation of the ACS seems not possible as all the ACS measurements reported by the Berciaud, Lounis and coworkers were in arbitrary units. ${ }^{13,14}$ In ref. 5, Gaiduk et al. overcame this problem by previously measuring the photothermal signal of a reference gold spherical nanoparticle of a given size, in the same environment, and computing the theoretical ACS using Mie theory. ${ }^{5}$ This gave them a calibration coefficient linking ACS and photothermal signal, assuming a linearity of the signal. Then, they performed measurements on single molecules, measured the photothermal signal, and used the calibration coefficient to indirectly retrieve an absolute value of the ACS of the molecule. The advantage of our technique is to perform a direct measurement of the ACS, in the sense that we do not need to perform a preliminary measurement on a model system (like a gold spherical NP) before investigating the absorbers of interest.

Other experimental approaches are based on the assumption that ACS can be assimilated to extinction cross section, like the approach reported by Arbouet et al.. ${ }^{3}$ An extinction cross section is indeed simpler to measure. This assumption is valid only for very small NPs when scattering is negligible, for example for gold NPs smaller than $30 \mathrm{~nm}$. Hence, such techniques cannot be applied, for instance, to larger structures or to extended distributions such as the ones reported in the present work.

Moreover, the QWLSI technique is straightforward to implement on any microscope, since one just has to use a CCD-based device. The use of an acousto-optic modulator, a single photon counting module, a heterodyne detection or to scan the sample to get an image, like the approaches listed above, is not required.

Finally, the QWLSI technique is faster than the above mentioned techniques. A entire image is obtained in less than 1 second while other PTI techniques require to scan an image with an integration time of approx. $10 \mathrm{~ms}$ to $200 \mathrm{~ms}$ per pixel. For this reason, our approach can be conveniently used to search and find out the resonance wavelength of an absorbing structure, in a reasonable time. The direct consequence is that our technique remains approximatively $10^{3}$ times less sensitive than other techniques that can detect single molecules. Our technique stands for this reason as a complementary technique compared to the existing field of techniques, a technique valuable when the ACS of interest are not too weak, typically for lithographic metal nanostructures larger than $100 \mathrm{~nm}$.

\section{CONCLUSION}

In summary, we recently introduced an optical microscopy technique able to map the distributions of temperature and heat power density around metal nanostructures. ${ }^{6}$ This technique is based on quadriwave lateral shearing interferometry (QWLSI). Here, we follow this development and detail how to utilize a similar experimental procedure to achieve quantitative absorption spectroscopy on single absorbers. At the current stage of its development, our approach can measure absorption cross sections as small as $10^{3} \mathrm{~nm}^{2}$. We decided to illustrate the technique on gold nanoparticles featuring plasmonics resonances. Different plasmonics modes were evidenced and quantified on a gold dimer. This sensitivity could be improved by modifying the liquid environment or using a faster CCD camera to collect more photons. We also evidenced that single NP absorption measurements can be affected by the absorption properties of the environment, especially when working under infrared illumination. The environment contribution can be minimized by adjusting geometrical and illumination parameters. This technique is straightforward to implement on any conventional microscope. The emerging field of thermo-plasmonics, which uses metal nanoparticles as nano-sources of heat, should benefit from this technique since the conception of highly absorbing nanostructures is still a subject of active investigations.
* pascal.berto@fresnel.fr

† guillaume.baffou@fresnel.fr

1 G. Baffou and R. Quidant, Laser \& Photon. Rev., DOI: 10.1002/lpor.201200003(2012).

2 A. O. Govorov and H. H. Richardson, Nano Today 2, 30 (2007).

3 A. Arbouet, D. Christofilos, N. Del Fatti, F. Vallée, J. R. Huntzinger, L. Arnaud, P. Billaud, and M. Broyer, Phys. Rev. Lett. 93, 127401 (2004).

4 M. Celebrano, P. Kukura, A. Renn, and V. Sandoghdar, Nature Photon. 5, 95 (2011).

5 A. Gaiduk, M. Yorulmaz, P. V. Ruijgrok, and M. Orrit, Science 330, 353 (2010).

6 G. Baffou, P. Bon, J. Savatier, J. Polleux, M. Zhu, M. Merlin, H. Rigneault, and S. Monneret, ACS Nano 6, 2452
(2012).

7 J. Primot and N. Guérineau, Appl. Opt. 39, 5715 (2000).

${ }^{8}$ G. Baffou, R. Quidant, and C. Girard, Phys. Rev. B 82, 165424 (2010).

9 P. Ghenuche, S. Cherukulappurath, T. H. Taminiau, N. F. van Hulst, and R. Quidant, Phys. Rev. Lett. 101, 116805 (2008).

10 P. K. Jain, K. S. Lee, I. H. El-Sayed, and M. A. El-Sayed, J. Phys. Chem. B 110, 7238 (2006), ISSN 1520-6106.

11 D. Boyer, P. Tamarat, A. Maali, B. Lounis, and M. Orrit, Science 297, 1160 (2002).

12 S. Berciaud, L. Cognet, G. A. Blab, and B. Lounis, Phys. Rev. Lett. 93, 257402 (2004).

13 S. Berciaud, L. Cognet, P. Poulin, R. B. Weisman, and B. Lounis, Nano Lett. 7, 1203 (2007). 
14 S. Berciaud, L. Cognet, and B. Lounis, Nano Lett. 5, 2160 (2005). 